\title{
Variaciones estacionales de las concentraciones de fosfatos y nitratos en distintas fuentes de aguas de pequeños productores hortícolas
}

\author{
Rodríguez, Silvia C. ${ }^{1}$; De Asmundis, Cecilia. L. "; Martínez, Gloria C. ${ }^{1}$ \\ ${ }^{1}$ Facultad de Ciencias Agrarias - Universidad Nacional del Nordeste \\ e-mail: silvicarlo@yahoo.com.ar
}

\begin{abstract}
RESUMEN
La agroindustria es una de las actividades productivas con gran responsabilidad en la contaminación de las aguas muchas veces debido al uso inadecuado de productos químicos. La calidad del agua se evalúa como una herramienta esencial para determinar la eficacia de las actividades de uso de la tierra y la gestión en su accionar agrícola, dada la presencia de elementos que pueden ocasionar problemas de contaminación tales como los nitratos, nutriente que más frecuentemente contamina los acuíferos y por otro lado la presencia de fósforo en el agua puede ser debida a los agroquímicos fosforados y al aporte de fertilizantes. En razón de la importancia que tienen estos iones en el agua de riego, en este trabajo se evaluaron las variaciones de las concentraciones de nitratos y fosfatos en las diferentes estaciones del año 2013, para distintas fuentes de agua, de pequeños productores hortícolas del NE de la provincia de Corrientes, realizándose muestreos, en agua superficial y en agua subterránea. Las determinaciones se realizaron por espectrometría de absorción UV-Visible. Los resultados muestran que hay un comportamiento opuesto de los analitos durante las distintas estaciones para el agua subterránea, no así para el agua superficial. Las concentraciones de ambos analitos en agua superficial presentan poca variación entre los distintos puntos de muestreos y son más bajos que los registrados en las aguas subterráneas mientras que las perforaciones y pozos presentan una alta variabilidad lo cual demuestra la independencia de los distintos puntos de muestreos y la expresión propia de cada fuente.
\end{abstract}

Palabras clave: fosfato, nitrato, agua superficial, agua subterránea.

\section{INTRODUCCIÓN}

En la actualidad, es importante conocer la calidad del agua tanto para el consumo humano como también para el riego de cultivos, la expedición de licencias ambientales, diseñar y ejecutar programas de monitoreo en las evaluaciones ambientales y adecuarla a las múltiples aplicaciones analíticas de los laboratorios, entre muchos otros fines (Cárdenas, 2003).

La agroindustria es una de las actividades productivas con gran responsabilidad en la contaminación de las aguas con productos químicos extremadamente peligrosos que pueden tener grandes efectos sobre la salud humana y la biota acuática en general, aún en cantidades muy reducidas. Muchos de estos agroquímicos simplemente no debieran ser utilizados, porque una vez que ingresan al ciclo hidrológico, se difunden a grandes distancias, son muy difíciles de detectar $\mathrm{y}$, por su presencia a nivel prácticamente molecular, no es posible filtrarlos o neutralizarlos una vez en el medio, provocando los efectos dañinos en el medio ambiente (Orrego, 2002).

La presencia de fosfatos en aguas potables indica la posibilidad de contaminación del acuífero por aguas contaminadas o aguas residuales. Debido a que el fósforo se encuentra presente en cantidades re-

Recibido: 08/07/2015. Aceptado: 25/11/2015 lativamente altas en aguas residuales y aguas de riego agrícola, su presencia en valores mayores a los valores normales, puede deberse a una contaminación o infiltración de aguas residuales al yacimiento de agua potable. Los herbicidas o pesticidas organofosforados que también están presentes en las aguas de riego agrícola son una advertencia de la calidad del agua ya que la presencia de fósforo en el agua puede ser debida a los agroquímicos fosforados. Si se debe a la infiltración de aguas residuales sin tratamiento previo, también son un riesgo al consumidor de estas fuentes de agua natural. En los últimos años, la presencia de fosfatos como contaminantes del agua ha adquirido mucha importancia debido a la escasez del recurso hídrico y a su deterioro creciente. Son sustancias solubles que las plantas necesitan para su desarrollo y que si se encuentran en el agua en cantidades excesivas inducen el crecimiento desmesurado de algas y otros organismos provocando procesos de polución y eutrofización. La eutrofización puede comenzar a partir de contenidos de fósforo tan bajos como $20 \mu \mathrm{g} \mathrm{L}^{-1}$, por lo que el lixiviado de fosfatos desde el suelo a los sistemas acuáticos supone, a menudo, serias consecuencias sobre los parámetros de los que depende la calidad medioambiental (Sande Fouz, 2002). Los fosfatos, constituyentes naturales de rocas y minerales, forman parte de fertilizantes 
y estiércoles, de la materia orgánica proveniente de desechos urbanos, industriales y residuos agrícolas como así también de los detergentes de uso industrial y doméstico. En muchos casos, estas fuentes de contaminación son arrastradas o arrojadas a las acequias, canales, arroyos, ríos y lagos, constituyendo un grave problema ambiental.

En general, la contaminación fosfatada es considerada una consecuencia directa del uso de fertilizantes por la agricultura; la mayoría de los estudios publicados sobre el tema plantea el problema de una agricultura intensiva, no respetuosa del medio ambiente (Lavie et al., 2010).

Actualmente se admite que existen, al menos, tres mecanismos de transferencia de fosfato desde el suelo a los sistemas acuáticos: disolución y movimiento en forma soluble; transporte de formas adsorbidas, asociado al desplazamiento vertical de la fracción coloidal y, sobre todo, a los materiales erosionados; y, por último, pérdidas directas de fertilizante cuando llueve tras la aplicación del mismo. La importancia de cada uno de estos mecanismos puede variar en función del tipo de suelo, del manejo agrícola y de las condiciones climáticas (Sande Fouz, 2002). El fosfato se encuentra en el agua en concentraciones muy bajas $\left(0,01 \mathrm{mg} \mathrm{L}^{-1}\right)$, lo que complica su determinación (Camacho Ferre, 2003).

El aprovechamiento de aguas residuales para riego agrícola podría en principio presuponer una menor exigencia que el necesario para uso industrial. Sin embargo, deben considerarse los riesgos asociados para la salud y el ambiente (Scott y Raschid, 2004) debido a la presencia de elementos que pueden ocasionar problemas de contaminación de aguas subterráneas (Morris et al., 2003), tales como los nitratos, nutriente que más comúnmente contamina los acuíferos (Muñoz, 2004).

El $60 \%$ del consumo energético de una explotación agrícola se debe a la síntesis del fertilizante mineral nitrogenado. Por lo que este proceso produce la mayor parte de las emisiones de gases de efecto invernadero en la agricultura; éste es el responsable del mayor porcentaje de producción de este gas. Por lo tanto, la implantación de las Buenas Practicas Agrarias (BPA) es la vía para reducir las emisiones de gases. Para ello se hace necesaria la supresión de determinados hábitos, como la quema de rastrojos, la quema de restos de poda, el laboreo excesivo y el manejo inadecuado de fertilizantes, tanto orgánicos como minerales (IDAE, 2007). Amoniaco, nitratos y nitritos son formas de nitrógeno presentes en los ecosistemas terrestres y acuáticos. De estas tres formas de nitrógeno presente en el agua de riego, los nitratos son los que ambientalmente suponen un mayor riesgo ya que, al igual que el fósforo, pueden causar problemas de eutrofización. Altos niveles de nitratos en aguas de riego se dan, principalmente, en aéreas de agricultura y ganadería intensivas (Bauder et al., 2008).

Los nitratos son compuestos químicos que en ocasiones se encuentran en el agua de pozos. Por lo general, los niveles elevados de nitratos en el agua extraída de pozos se deben a la contaminación en las aguas subterráneas por el uso excesivo de fertilizantes, los residuos de granjas mal gestionados, o la infiltración de drenaje humano proveniente de las fosas sépticas (Castiella, et al., 2008).

Dada la relación entre el riego y el movimiento de los nitratos en el suelo, la eficiencia en el uso del agua de irrigación y del nitrógeno aplicado por fertilización adquiere gran relevancia, si se pretende realizar una producción sustentable. Las distintas textura de los suelos condicionan el movimiento del agua del suelo, generando en algunos casos condiciones favorables para la migración de los nitratos en profundidad (Rimski-Korsakov et al., 2002).

El objetivo de este trabajo fue evaluar las variaciones de las concentraciones de fosfatos y nitratos en las diferentes estaciones del año, para distintas fuentes de agua, de pequeños productores hortícolas.

\section{MATERIALES Y MÉTODOS}

El trabajo se realizó en parcelas productivas de pequeños productores hortícolas del Departamento de Itatí, provincia de Corrientes, Argentina que se caracteriza por presentar clima subtropical húmedo, con veranos calurosos y abundantes lluvias. La precipitación media anual es de $1200 \mathrm{~mm}$.

Se realizó un relevamiento de la zona de estudio con el fin de establecer cuál era la fuente de agua que estos pequeños productores utilizaban tanto para el riego como para consumo humano y animal, la cercanía de estas a corrales, estercoleros y las distancias de pozos sépticos.

Se seleccionaron las fuentes de agua que los mismos utilizan para riego y otros usos; siendo éstas fuentes una laguna (agua superficial) con cuatro puntos de muestreo en función de la cercanía a las chacras; cuatro pozos a balde y cuatro perforaciones (agua subterránea). En el caso de la laguna también es utilizada como bebedero de animales.

Los lugares de muestreo pertenecen a zonas rurales que no reciben aguas residuales, ya que se encuentran alejados del principal núcleo urbano que es la localidad de Itatí.

Se realizaron muestreos mensuales y en las distintas estaciones del año para cada fuente de agua (agua superficial y agua subterránea); desde enero a diciembre del año 2013.

La toma de las muestras para análisis físico-químico se realizó del siguiente modo (Rodríguez et al., 2012). 


\section{Para agua de perforación:}

La muestra se tomó de la cañería inmediata a la perforación, manteniendo la marcha de la impulsión un tiempo; en el caso de perforaciones nuevas se bombeó el tiempo suficiente hasta que el agua salió limpia, de manera de muestrear el agua del acuífero.

\section{Para agua de pozo a balde:}

Se bajó el balde dentro del pozo hasta una profundidad de 15 a $30 \mathrm{~cm}$. desde la superficie libre del líquido, evitando en todo momento tocar las paredes del pozo. Luego se llenó la botella directamente con el agua del balde.

\section{Para agua de laguna:}

Se utilizó una pinza de brazos largos para no muestrear del borde. Evitando tomar la muestra de la capa superficial, se sumergió la botella en el agua con un peso incorporado, a una profundidad de 15 a $30 \mathrm{~cm}$. Se retiró la botella después que no se observó ascenso de burbujas.

En todos los casos, se tomó la precaución de enjuagar el recipiente de muestreo, 3 veces con la misma agua muestreada, desechando el agua de enjuague; se recogió la muestra tratando de no dejar cámara de aire, aunque se puede dejar un mínimo sin llenar que permita la variación de volumen debida a potenciales diferencias térmicas.

Se determinó In Situ temperatura, pH y Conductividad eléctrica de la muestra.

Se cerró herméticamente el envase. Se guardó la muestra en lugar fresco y se trasladó al Laboratorio en el menor tiempo posible.

\section{Análisis físicos-químicos:}

Determinación de Nitrato: aplicando el método de salicilato de sodio por Espectrofotometría de Absorción molecular (Rodríguez et al., 2005).

Determinación de Fosfato: por Espectrofotometría de Absorción molecular: método del azul de molibdeno (APHA, 2005).

Los datos fueron sometidos a un análisis de estadística descriptiva, regresión lineal simple, ANOVA y Test de Tukey (Infostat, 2014).

\section{RESULTADOS}

Para el fosfato se registraron los valores más altos en el invierno, para el agua subterránea y en el verano para el agua superficial; estos resultados de mayor concentración en agua superficial durante el verano es coincidente con lo encontrado por Sande Fouz (2002), quien registró los valores más altos de fosfatos en agua en estaciones del año donde el riesgo de erosión es mayor. Al igual que este último autor, Mazzeo et al., (2001) encontraron que la concentración de fósforo total fue en general baja, excepto en el verano.

En condiciones normales las concentraciones de los iones de nitrato en agua natural generalmente es baja, tal como sucedió en este trabajo. Los mayores valores de este analito fueron obtenidos en primavera y verano. Mazzeo et al., (2001) observaron que la concentración de nitrógeno total fue baja a moderada dependiendo del nivel de agua. No obstante Auge (1997) detectó que en las aguas subterráneas se observa con frecuencia una elevación de las concentraciones, como consecuencia de la intensificación de las prácticas agrícolas.

De los análisis estadísticos efectuados en los dos analitos, no se registraron interacciones entre fuentes y estaciones del año.

El contenido de fosfato en el agua superficial (laguna) registró valores promedios más bajos $(0,0016$ $\left.\mathrm{mg} \mathrm{L}^{-1}\right)$ difiriendo significativamente $(\alpha=0,05)$ del resto de las fuentes, las demás no mostraron diferencias entre ellas, con registros promedios de $0,017 \mathrm{y}$ $0,029 \mathrm{mg} \mathrm{L}^{-1}$ en perforación y pozo respectivamente.

El comportamiento de las concentraciones de este analito varió entre las distintas fuentes, en el caso del agua superficial los mayores registros fueron obtenidos en el verano y los menores durante el otoño, por el contrario en las aguas subterráneas se registraron los valores más altos en el invierno y primavera.

Para el agua superficial los registros de fosfato más bajos fueron obtenidos en el otoño $\left(0,0005 \mathrm{mg} \mathrm{L}^{-1}\right)$, si bien los valores más altos no superaron los 0,0022 $\mathrm{mg} \mathrm{L}^{-1}$ este valor obtenido fue durante el período de verano, en coincidencia con precipitaciones intensas y periódicas, lo que podría arrastrar componentes de suelo con compuestos de fosfatos a esta fuente. $\mathrm{Si}$ bien no es tan notoria la variación entre los datos de las distintas estaciones $(\mathrm{CV}=59,25)$, las diferencias entre ellas no son significativas (Figura $\mathrm{N}^{\circ} 1$ ).

El comportamiento de las concentraciones de fosfato obtenidos en las fuentes de agua subterránea fueron similares, con valores más altos durante el período de invierno $\left(0,020\right.$ y $0,033 \mathrm{mg} \mathrm{L}^{-1}$ para perforación y pozo respectivamente), y los registros más bajos se obtuvieron en el otoño para el agua de perforación $\left(0,014 \mathrm{mg} \mathrm{L}^{-1}\right)$ y en verano para el agua de pozo $\left(0,019 \mathrm{mg} \mathrm{L}^{-1}\right)$. Si bien existe una diferencia entre $28 \%$ y $42 \%$ entre los valores máximos y mínimos, para perforación y pozo respectivamente, la misma no alcanzan a ser significativas por la variabilidad ( $\mathrm{CV}=128$ y 129 respectivamente) de los datos de cada una de ellas (Figura $\mathrm{N}^{\circ} 2$ ).

En cuanto al nitrato el menor registro significativo de las concentraciones de este analito fue el obtenido en agua superficial con un valor promedio de todas las estaciones del año de $0,77 \mathrm{mg} \mathrm{L}^{-1}$. Sin embargo no se encontró diferencias entre las demás fuentes, siendo en el caso de perforación el registro obtenido de $5,79 \mathrm{mg} \mathrm{L}^{-1}$ y el más alto fue para el agua de pozo alcanzando un valor promedio de $8,11 \mathrm{mg} \mathrm{L}^{-1}$. 


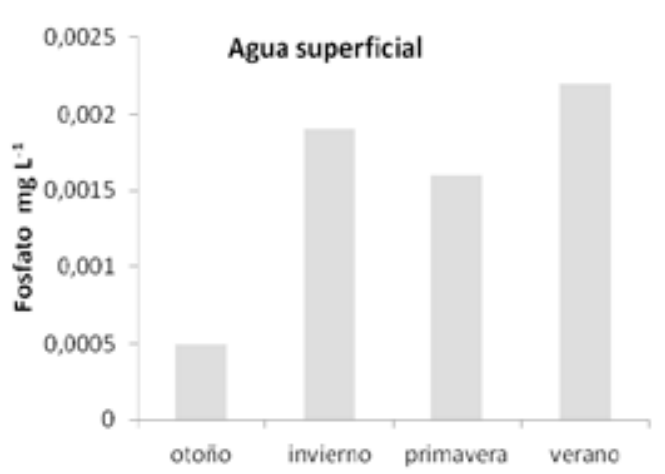

Figura 1. Concentración de Fosfato en $\mathrm{mg} \mathrm{L}^{-1}$ en las distintas estaciones del año para el agua superficial: Laguna.

En la Figura 3 se observa las concentraciones de nitratos para agua superficial (Laguna), las mismas se mantuvieron dentro de un rango de 0,64 a 0,88 $\mathrm{mg} \mathrm{L}^{-1}$. Los mayores registro se dieron en el otoño y verano $\left(0,82 \mathrm{mg} \mathrm{L}^{-1}\right.$ y $\left.0,88 \mathrm{mg} \mathrm{L}^{-1}\right)$, disminuyendo en invierno, con los registros más bajos en primavera; lo cual se podría deber a las lluvias registrada en este periodo.

Con respecto al contenido de nitrato en el agua subterránea (Figura 4), se observaron registros menores durante el otoño y el invierno, y mayores concentraciones en el verano y primavera. El agua de perforación no superó concentraciones de 7,11 mg $\mathrm{L}^{-1}$. Si bien se observa una diferencia de $65 \%$ de las concentraciones entre los registros más altos y más bajos, en las distintas estaciones del año, para esta fuente, no existieron diferencias significativas entre las mismas, lo que se debería a la gran variabilidad de los registros en los distintos lugares de muestreo. El agua de pozo presentó los mayores registros de nitrato, con respecto a las demás fuentes de agua, al igual que el agua de perforación, la variabilidad entre los lugares de muestreo fue notoria $(\mathrm{CV}=118)$. El comportamiento de las concentraciones fue similar a

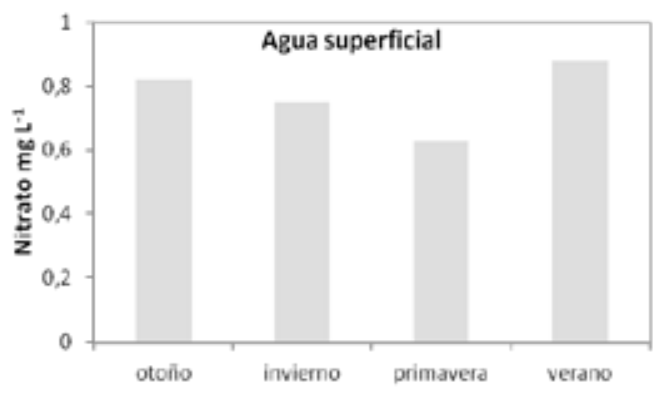

Figura 3. Concentración de Nitrato en $\mathrm{mg} \mathrm{L}^{-1}$ en las distintas estaciones del año para el agua superficial: Laguna.

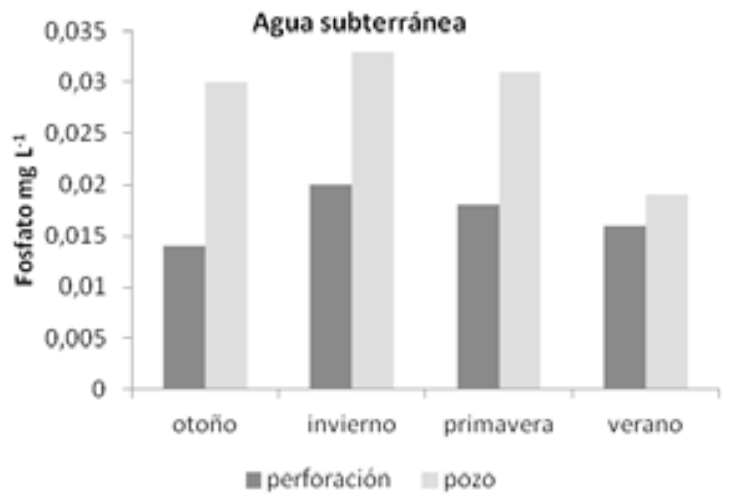

Figura 2. Concentración de Fosfato en $\mathrm{mg} \mathrm{L}^{-1}$ en las distintas estaciones del año para el agua subterránea: Perforación y Pozo.

lo observado en el agua de perforación, registrándose valores más altos en el verano $\left(14 \mathrm{mg} \mathrm{L}^{-1}\right)$ y durante el invierno se detectó una diferencia, con respecto a esta estación del $62 \%$ menos de las concentraciones de $\mathrm{NO}_{3}{ }^{-}$, sin ser significativo los registros entre estaciones.

Los mayores registros de concentraciones de $\mathrm{NO}_{3}^{-}$ fueron para el agua de pozo, lo cual podría deberse a la contaminación de esta fuente por infiltración de agua externa.

\section{CONCLUSIONES}

Se detectó un comportamiento opuesto de los analitos durante las distintas estaciones, en agua subterránea; encontrándose los valores de concentración más altos para fosfato en invierno y para nitrato en el verano, no así para el agua superficial, en la que los valores más altos de concentraciones de ambos analitos se encontraron en el verano.

La concentración tanto de nitratos como la de fosfatos en agua superficial son más bajos que las registradas en las aguas subterráneas y con poca variación entre los distintos puntos de muestreos.

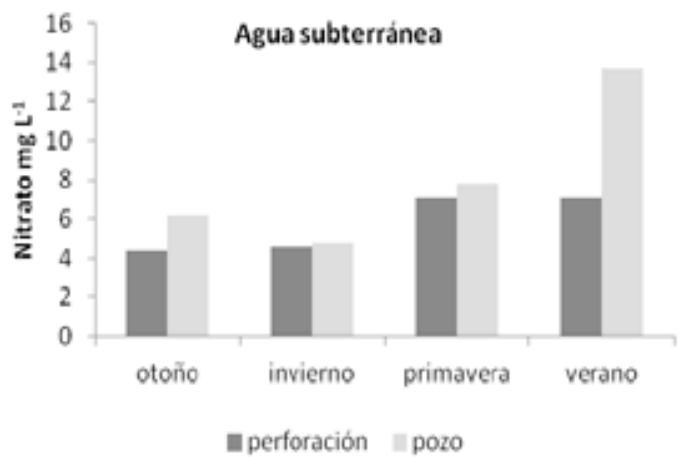

Figura 4. Concentración de Nitrato en $\mathrm{mg} \mathrm{L}^{-1}$ en las distintas estaciones del año para el agua subterránea: Perforación y Pozo. 
Los resultados obtenidos para perforaciones y pozo presentan una alta variabilidad lo cual demuestra la independencia de los distintos puntos de muestreos y la expresión propia de cada fuente.

\section{BIBLIOGRAFÍA}

American Public Health Association (APHA). American Water WorksAssociation and Water Pollution Control Federation. 2005. Standard Methods for the Examination of Water and Wastewater, 17 th ed., Washington, D.C.USA. Parte 9000.

Auge, M. 1997. Deterioro de acuíferos por sobreexplotación y contaminación. In: Congreso Internacional sobre Aguas, Conferencia Plenaria. Ed. Universidad de Buenos Aires. Buenos Aires, Argentina.

Bauder, T.A.; Waskom, R.M.; Davis, J.G. 2007. Irrigation Water Quality Criteria. Colorado State University Cooperative Extension (USA). 7/03. no.0.506. www.ext.colostate.edu

Camacho Ferre, F. 2003. Tecnicas de produccion en cultivos protegidos. Edita la Caja Rural Intermediterranea (Cajamar). Almeria (Espana). 373 páginas.

Cárdenas, J. 2003. La calidad de las aguas para estudiantes de Ciencias Ambientales. Universidad Distrital, Colombia.

Castiella, J.; Perez, C.; Sanz L. 2008. Documento técnico. Contaminación por nitratos en el Acuífero Aluvial del Ebro en Navarra, Dirección Gral. De Medio Ambiente y Agua V.1.0, CRANA (Fundación Centro de Recursos Ambientales de Navarra). Pamplona / Iruña.

IDAE (Instituto para la Diversificación y Ahorro de la Energía). 2007. Biomasa: Gasificación. ISBN-13: 97884-96680-20-3.

Infostat/P. 2014. Versión 1.1. Universidad Nacional de Córdoba, República Argentina. Estadística y Diseño -FCA.

Lavie, E; Bermejillo, A.; Morábito, J.: Filippini, M.; Salatino, S. 2010. Contaminación por fosfatos en el oasis bajo riego del río Mendoza. Rev. FCA UNCuyo. Tomo 42. $\mathrm{N}^{\circ} 1: 169-184$.

Mazzeo, N.; Kruk, C.; Meerhoff, M.; Quintans, F.; Lacerot, G.; Gorga, J.; Rodríguez, L.; Loureiro, M.; García, F.; Scharf, B.; Larrea, D. 2001. Evaluación de la calidad del agua de la Laguna Blanca: sus causas y respuestas. Financiamiento: Aguas de la Costa y CSIC (programa de vinculación al sector productivo) Montevideo, Uruguay.

Morris, B.L.; Laurence, A.R.L.; Chilton, P.J.C.; Adams, B.; Calow, R.C.; Klinck, B.A. 2003. Groundwater lands its susceptibility to degradation: A global assessment of the problem and options for management. Early warning and assessment report series, RS $03-3$. Unites Nations Environment Programme. Nairobi, Kenia.

Muñoz, H.; Armienta, A.; Vera, A.; Ceniceros, N. 2004. Nitrato en el agua subterránea del valle de Huamantla, Tlaxcala. Rev. Int. Contam. Ambie. 20, 91-97.
Orrego, J. 2002. El estado de las aguas terrestres en Chile: cursos y aguas subterráneas. Fundación Terram. Santiago, Chile.

Rimski-Korsakov, H.; Torres Duggan, M.; Lavado, R. S. 2002. Influencia de la Fertilización y el Riego en la Lixiviación de Nitratos en un Suelo Arenoso. XVII Congreso Argentino de la Ciencia del Suelo.

Rodríguez, S.C.; Fernández, J.A.; Martínez, G. 2005. Validación Interna de un método para la Determinación de Nitratos en Agua. XX Congreso Nacional del Agua. III Simposio de Recursos Hídricos del Cono Sur. Provincia de Mendoza. República Argentina.

Rodríguez, S.C.; Acevedo, H.; De Asmundis, C.; Martínez, G. 2012. Clasificación salina y sódico de agua de zonas hortícolas. En fuentes de agua subterráneas, de la Provincia de Corrientes, República Argentina. Editorial Académica Española (eae). ISBN: 978-3-8473-6073-5

Sande Fouz, P. 2002. Estudio del contenido en fósforo y sólidos en suspensión de aguas superficiales en pequeñas cuencas. Tesis de Licenciatura. Facultad de Ciencias. Universidad de La Coruña. 148 pp.

Scout, Ch. y Raschid, L. 2004. Waste water use in irrigated agriculture. Confronting the livelihood and environmental realities. CAB International. 